\title{
From Non-Adaptive to Adaptive Pseudorandom Functions
}

\author{
Itay Berman Iftach Haitner*
}

January 11, 2012

\begin{abstract}
Unlike the standard notion of pseudorandom functions (PRF), a non-adaptive PRF is only required to be indistinguishable from a random function in the eyes of a non-adaptive distinguisher (i.e., one that prepares its oracle calls in advance). A recent line of research has studied the possibility of a direct construction of adaptive PRFs from non-adaptive ones, where direct means that the constructed adaptive PRF uses only few (ideally, constant number of) calls to the underlying non-adaptive PRF. Unfortunately, this study has only yielded negative results, showing that "natural" such constructions are unlikely to exist (e.g., Myers [EUROCRYPT '04], Pietrzak [CRYPTO '05, EUROCRYPT '06]).

We give an affirmative answer to the above question, presenting a direct construction of adaptive PRFs from non-adaptive ones. The suggested construction is extremely simple, a composition of the non-adaptive PRF with an appropriate pairwise independent hash function.
\end{abstract}

\section{Introduction}

A pseudorandom function family (PRF), introduced by Goldreich, Goldwasser, and Micali [11], cannot be distinguished from a family of truly random functions by an efficient distinguisher who is given an oracle access to a random member of the family. PRFs have an extremely important role in cryptography, allowing parties, which share a common secret key, to send secure messages, identify themselves and to authenticate messages [10, 13]. In addition, they have many other applications, essentially in any setting that requires random function provided as black-box $[2,3$, $6,7,14,18]$. Different PRF constructions are known in the literature, whose security is based on different hardness assumption. Constructions relevant to this work are those based on the existence of pseudorandom generators [11] (and thus on the existence of one-way functions [12]), and on, the so called, synthesizers [17].

In this work we study the question of constructing (adaptive) PRFs from non-adaptive PRFs. The latter primitive is a (weaker) variant of the standard PRF we mentioned above, whose security is only guaranteed to hold against non-adaptive distinguishers (i.e., ones that "write" all their queries before the first oracle call). Since a non-adaptive PRF can be easily cast as a pseudorandom generator or as a synthesizer, $[11,17]$ tell us how to construct (adaptive) PRF from a non-adaptive one. In both of these constructions, however, the resulting (adaptive) PRF makes $\Theta(n)$ calls to the underlying non-adaptive PRF (where $n$ being the input length of the functions). ${ }^{1}$

\footnotetext{
${ }^{*}$ School of Computer Science, Tel Aviv University. E-mail: iftachh@cs.tau.ac.il, itayberm@post.tau.ac.il.

${ }^{1}$ We remark that if one is only interested in polynomial security (i.e., no adaptive PPT distinguishes with more than negligible probability), then $w(\log n)$ calls are sufficient (cf., [8, Sec. 3.8.4, Exe. 30]).
} 
A recent line of work has tried to figure out whether more efficient reductions from adaptive to non-adaptive PRF's are likely to exist. In a sequence of works $[16,19,20,5]$, it was shown that several "natural" approaches (e.g., composition or XORing members of the non-adaptive family with itself) are unlikely to work. See more in Section 1.3.

\section{$1.1 \quad$ Our Result}

We show that a simple composition of a non-adaptive PRF with an appropriate pairwise independent hash function, yields an adaptive PRF. To state our result more formally, we use the following definitions: a function family $\mathcal{F}$ is $T=T(n)$-adaptive PRF, if no distinguisher of running time at most $T$, can tell a random member of $\mathcal{F}$ from a random function with advantage larger than $1 / T$. The family $\mathcal{F}$ is $T$-non-adaptive PRF, if the above is only guarantee to hold against non-adaptive distinguishers. Given two function families $\mathcal{F}_{1}$ and $\mathcal{F}_{2}$, we let $\mathcal{F}_{1} \circ \mathcal{F}_{2}$ [resp., $\mathcal{F}_{1} \bigoplus \mathcal{F}_{2}$ ] be the function family whose members are all pairs $(f, g) \in \mathcal{F}_{1} \times \mathcal{F}_{2}$, and the action $(f, g)(x)$ is defined as $f(g(x))$ [resp., $f(x) \oplus g(x)$ ]. We prove the following statements (see Section 3 for the formal statements).

Theorem 1.1 (Informal). Let $\mathcal{F}$ be $a(p(n) \cdot T(n))$-non-adaptive PRF, where $p \in$ poly is function of the evaluating time of $\mathcal{F}$, and let $\mathcal{H}$ be an efficient pairwise-independent function family mapping strings of length $n$ to $[T(n)]_{\{0,1\}^{n}}$, where $[T]_{\{0,1\}^{n}}$ is the first $T$ elements (in lexicographic order) of $\{0,1\}^{n}$. Then $\mathcal{F} \circ \mathcal{H}$ is a $(\sqrt[3]{T(n)} / 2)$-adaptive PRF.

For instance, assuming that $\mathcal{F}$ is a $\left(p(n) \cdot 2^{c n}\right)$-non-adaptive PRF and that $\mathcal{H}$ maps strings of length $n$ to $\left[2^{c n}\right]_{\{0,1\}^{n}}$, Theorem 1.1 yields that $\mathcal{F} \circ \mathcal{H}$ is a $\left(2^{\frac{c n}{3}-1}\right)$-adaptive PRF.

Theorem 1.1 is only useful, however, for polynomial-time computable $T$ 's (in this case, the family $\mathcal{H}$ assumed by the theorem exists, see Section 2.2.2). Unfortunately, in the important case where $\mathcal{F}$ is only assumed to be polynomially secure non-adaptive PRF, no useful polynomial-time computable $T$ is guaranteed to exists. ${ }^{2}$

We suggest two different solutions for handling polynomially secure PRFs. In Appendix A we observe (following Bellare [1]) that a polynomially secure non-adaptive PRF is a $T$-non-adaptive PRF for some $T \in n^{\omega(1)}$. Since this $T$ can be assumed without loss of generality to be a power of two, Theorem 1.1 yields a non-uniform (uses $n$-bit advice) polynomially secure adaptive PRF, that makes a single call to the underlying non-adaptive PRF. Our second solution is to use the following "combiner", to construct a (uniform) adaptively secure PRF, which makes $\omega(1)$ parallel calls to the underlying non-adaptive PRF.

Corollary 1.2 (Informal). Let $\mathcal{F}$ be a polynomially secure non-adaptive PRF, let $\mathcal{H}=\left\{\mathcal{H}_{n}\right\}_{n \in \mathbb{N}}$ be an efficient pairwise-independent length-preserving function family and let $k(n) \in \omega(1)$ be polynomial-time computable function.

For $n \in \mathbb{N}$ and $i \in[n]$, let $\widehat{\mathcal{H}}_{n}^{i}$ be the function family $\widehat{\mathcal{H}}_{n}^{i}=\{\widehat{h}: h \in \mathcal{H}\}$, where $\widehat{h}(x)=0^{n-i} \| h(x)_{1, \ldots, i}$ (' $\|$ ' stands for string concatenation). Then the ensemble $\left\{\bigoplus_{i \in[k(n)]}\left(\mathcal{F}_{n} \circ \widehat{\mathcal{H}}_{n}^{\lfloor i \cdot \log n\rfloor}\right)\right\}_{n \in \mathbb{N}}$ is a polynomially secure adaptive PRF.

\footnotetext{
${ }^{2}$ Clearly $\mathcal{F}$ is $p$-non-adaptive PRF for any $p \in$ poly, but applying Theorem 1.1 with $T \in$ poly, does not yield a polynomially secure adaptive PRF.
} 


\subsection{Proof Idea}

To prove Theorem 1.1 we first show that $\mathcal{F} \circ \mathcal{H}$ is indistinguishable from $\Pi \circ \mathcal{H}$, where $\Pi$ being the set of all functions from $\{0,1\}^{n}$ to $\{0,1\}^{\ell(n)}$ (letting $\ell(n)$ be $\mathcal{F}^{\prime}$ 's output length), and then conclude the proof by showing that $\Pi \circ \mathcal{H}$ is indistinguishable from $\Pi$.

$\mathcal{F} \circ \mathcal{H}$ is indistinguishable from $\Pi \circ \mathcal{H}$. Let $\mathrm{D}$ be (a possibly adaptive) algorithm of running time $T(n)$, which distinguishes $\mathcal{F} \circ \mathcal{H}$ from $\Pi \circ \mathcal{H}$ with advantage $\varepsilon(n)$. We use $\mathrm{D}$ to build a non-adaptive distinguisher $\widehat{\mathrm{D}}$ of running time $p(n) \cdot T(n)$, which distinguishes $\mathcal{F}$ from $\Pi$ with advantage $\varepsilon(n)$. Given an oracle access to a function $\phi$, the distinguisher $\widehat{\mathrm{D}}^{\phi}\left(1^{n}\right)$ first queries $\phi$ on all the elements of $[T(n)]_{\{0,1\}^{n}}$. Next it chooses at uniform $h \in \mathcal{H}$, and uses the stored answers to its queries, to emulate $\mathrm{D}^{\phi \circ h}\left(1^{n}\right)$.

Since $\widehat{D}$ runs in time $p(n) \cdot T(n)$, for some large enough $p \in$ poly, makes non-adaptive queries, and distinguishes $\mathcal{F}$ from $\Pi$ with advantage $\varepsilon(n)$, the assumed security of $\mathcal{F}$ yields that $\varepsilon(n)<\frac{1}{p(n) \cdot T(n)}$.

$\Pi \circ \mathcal{H}$ is indistinguishable from $\Pi$. We prove that $\Pi \circ \mathcal{H}$ is statistically indistinguishable from $\Pi$. Namely, even an unbounded distinguisher (that makes bounded number of calls) cannot distinguish between the families. The idea of the proof is fairly simple. Let $\mathrm{D}$ be an $s$-query algorithm trying to distinguish between $\Pi \circ \mathcal{H}$ and $\Pi$. We first note that the distinguishing advantage of $\mathrm{D}$ is bounded by its probability of finding a collision in a random $\phi \in \Pi \circ \mathcal{H}$ (in case no collision occurs, $\phi$ 's output is uniform). We next argue that in order to find a collision in $\phi$, the distinguisher $\mathrm{D}$ gains nothing from being adaptive. Indeed, assuming that $\mathrm{D}$ found no collision until the $i$ 'th call, then it has only learned that $h$ does not collide on these first $i$ queries. Therefore, a random (or even a constant) query as the $(i+1)$ call, has the same chance to yield a collision, as any other query has. Hence, we assume without loss of generality that $\mathrm{D}$ is non-adaptive, and use the pairwise independence of $\mathcal{H}$ to conclude that D's probability in finding a collision, and thus its distinguishing advantage, is bounded by $s(n)^{2} / T(n)$.

Combining the above two observations, we conclude that an adaptive distinguisher whose running time is bounded by $\frac{1}{2} \sqrt[3]{T(n)}$, cannot distinguish $\mathcal{F} \circ \mathcal{H}$ from $\Pi$ (i.e., from a random function) with

an advantage better than $\frac{T(n)^{\frac{2}{3}} / 4}{T(n)}+\frac{1}{p(n) T(n)} \leq 2 / \sqrt[3]{T(n)}$. Namely, $\mathcal{F} \circ \mathcal{H}$ is a $(\sqrt[3]{T(n)} / 2)$-adaptive PRF.

\subsection{Related Work}

Maurer and Pietrzak [15] were the first to consider the question of building adaptive PRFs from non-adaptive ones. They showed that in the information theoretic model, a self composition of a non-adaptive PRF does yield an adaptive PRF. ${ }^{3}$

In contrast, the situation in the computational model (which we consider here) seems very different: Myers [16] proved that it is impossible to reprove the result of [15] via fully-black-box reductions. Pietrzak [19] showed that under the Decisional Diffie-Hellman (DDH) assumption,

\footnotetext{
${ }^{3}$ Specifically, assuming that the non-adaptive $\mathrm{PRF}$ is $(Q, \varepsilon)$-non-adaptively secure, no $Q$-query non-adaptive algorithm distinguishes it from random with advantage larger than $\varepsilon$, then the resulting $\operatorname{PRF}$ is $\left(Q, \varepsilon\left(1+\ln \frac{1}{\varepsilon}\right)\right)$-adaptively secure.
} 
composition does not imply adaptive security. Where in [20] he showed that the existence of nonadaptive PRFs whose composition is not adaptively secure, yields that key-agreement protocol exists. Finally, Cho et al. [5] generalized [20] by proving that composition of two non-adaptive PRFs is not adaptively secure, iff (uniform transcript) key agreement protocol exists. We mention that $[16,19,5]$, and in a sense also [15], hold also with respect to XORing of the non-adaptive families.

\section{Preliminaries}

\section{$2.1 \quad$ Notations}

All logarithms considered here are in base two. We let 'Il' denote string concatenation. We use calligraphic letters to denote sets, uppercase for random variables, and lowercase for values. For an integer $t$, we let $[t]=\{1, \ldots, t\}$, and for a set $\mathcal{S} \subseteq\{0,1\}^{*}$ with $|\mathcal{S}| \geq t$, we let $[t]_{\mathcal{S}}$ be the first $t$ elements (in increasing lexicographic order) of $\mathcal{S}$. A function $\mu: \mathbb{N} \rightarrow[0,1]$ is negligible, denoted $\mu(n)=\operatorname{neg}(n)$, if $\mu(n)=n^{-\omega(1)}$. We let poly denote the set all polynomials, and let PPT denote the set of probabilistic algorithms (i.e., Turing machines) that run in strictly polynomial time.

Given a random variable $X$, we write $X(x)$ to denote $\operatorname{Pr}[X=x]$, and write $x \leftarrow X$ to indicate that $x$ is selected according to $X$. Similarly, given a finite set $\mathcal{S}$, we let $s \leftarrow \mathcal{S}$ denote that $s$ is selected according to the uniform distribution on $\mathcal{S}$. The statistical distance of two distributions $P$ and $Q$ over a finite set $\mathcal{U}$, denoted as $\operatorname{SD}(P, Q)$, is defined as $\max _{\mathcal{S} \subseteq \mathcal{U}}|P(\mathcal{S})-Q(\mathcal{S})|=$ $\frac{1}{2} \sum_{u \in \mathcal{U}}|P(u)-Q(u)|$.

\subsection{Ensemble of Function Families}

Let $\mathcal{F}=\left\{\mathcal{F}_{n}: \mathcal{D}_{n} \mapsto \mathcal{R}_{n}\right\}_{n \in \mathbb{N}}$ stands for an ensemble of function families, where each $f \in \mathcal{F}_{n}$ has domain $\mathcal{D}_{n}$ and its range contained in $\mathcal{R}_{n}$. Such ensemble is length preserving, if $\mathcal{D}_{n}=\mathcal{R}_{n}=\{0,1\}^{n}$ for every $n$.

Definition 2.1 (efficient function family ensembles). A function family ensemble $\mathcal{F}=\left\{\mathcal{F}_{n}\right\}_{n \in \mathbb{N}}$ is efficient, if the following hold:

Samplable. $\mathcal{F}$ is samplable in polynomial-time: there exists a PPT that given $1^{n}$, outputs (the description of) a uniform element in $\mathcal{F}_{n}$.

Efficient. There exists a polynomial-time algorithm that given $x \in\{0,1\}^{n}$ and (a description of) $f \in \mathcal{F}_{n}$, outputs $f(x)$.

\subsubsection{Operating on Function Families}

Definition 2.2 (composition of function families). Let $\mathcal{F}^{1}=\left\{\mathcal{F}_{n}^{1}: \mathcal{D}_{n}^{1} \mapsto \mathcal{R}_{n}^{1}\right\}_{n \in \mathbb{N}}$ and $\mathcal{F}^{2}=$ $\left\{\mathcal{F}_{n}^{2}: \mathcal{D}_{n}^{2} \mapsto \mathcal{R}_{n}^{2}\right\}_{n \in \mathbb{N}}$ be two ensembles of function families with $\mathcal{R}_{n}^{1} \subseteq \mathcal{D}_{n}^{2}$ for every $n$. We define the composition of $\mathcal{F}^{1}$ with $\mathcal{F}^{2}$ as $\mathcal{F}^{2} \circ \mathcal{F}^{1}=\left\{\mathcal{F}_{n}^{2} \circ \mathcal{F}_{n}^{1}: \mathcal{D}_{n}^{1} \mapsto \mathcal{R}_{n}^{2}\right\}_{n \in \mathbb{N}}$, where $\mathcal{F}_{n}^{2} \circ \mathcal{F}_{n}^{1}=\left\{\left(f_{2}, f_{1}\right) \in\right.$ $\left.\mathcal{F}_{n}^{2} \times \mathcal{F}_{n}^{1}\right\}$, and $\left(f_{2}, f_{1}\right)(x):=f_{2}\left(f_{1}(x)\right)$.

Definition 2.3 (XOR of function families). Let $\mathcal{F}^{1}=\left\{\mathcal{F}_{n}^{1}: \mathcal{D}_{n}^{1} \mapsto \mathcal{R}_{n}^{1}\right\}_{n \in \mathbb{N}}$ and $\mathcal{F}^{2}=\left\{\mathcal{F}_{n}^{2}: \mathcal{D}_{n}^{2} \mapsto\right.$ $\left.\mathcal{R}_{n}^{2}\right\}_{n \in \mathbb{N}}$ be two ensembles of function families with $\mathcal{R}_{n}^{1}, \mathcal{R}_{n}^{2} \subseteq\{0,1\}^{\ell(n)}$ for every $n$. We define the XOR of $\mathcal{F}^{1}$ with $\mathcal{F}^{2}$ as $\mathcal{F}^{2} \bigoplus \mathcal{F}^{1}=\left\{\mathcal{F}_{n}^{2} \oplus \mathcal{F}_{n}^{1}: \mathcal{D}_{n}^{1} \cap \mathcal{D}_{n}^{2} \mapsto\{0,1\}^{\ell(n)}\right\}_{n \in \mathbb{N}}$, where $\mathcal{F}_{n}^{2} \bigoplus \mathcal{F}_{n}^{1}=$ $\left\{\left(f_{2}, f_{1}\right) \in \mathcal{F}_{n}^{2} \times \mathcal{F}_{n}^{1}\right\}$, and $\left(f_{2}, f_{1}\right)(x):=f_{2}(x) \oplus f_{1}(x)$. 


\subsubsection{Pairwise Independent Hashing}

Definition 2.4 (pairwise independent families). A function family $\mathcal{H}=\{h: \mathcal{D} \mapsto \mathcal{R}\}$ is pairwise independent (with respect to $\mathcal{D}$ and $\mathcal{R}$ ), if

$$
\operatorname{Pr}_{h \leftarrow \mathcal{H}}\left[h\left(x_{1}\right)=y_{1} \wedge h\left(x_{2}\right)=y_{2}\right]=\frac{1}{|\mathcal{R}|^{2}}
$$

for every distinct $x_{1}, x_{2} \in \mathcal{D}$ and every $y_{1}, y_{2} \in \mathcal{R}$.

For every $\ell \in$ poly, the existence of efficient pairwise-independent family ensembles mapping strings of length $n$ to strings of length $\ell(n)$ is well known ([4]). In this paper we use efficient pairwiseindependent function family ensembles mapping strings of length $n$ to the set $[T(n)]_{\{0,1\}^{n}}$, where $T(n) \leq 2^{n}$ and is without loss of generality a power of two. ${ }^{4}$ Let $\mathcal{H}$ be an efficient length-preserving, pairwise-independent function family ensemble and assume that $t(n):=\log T(n)$ is polynomial-time computable. Then the function family $\widehat{\mathcal{H}}=\left\{\widehat{\mathcal{H}_{n}}=\left\{h^{\prime}: h \in \mathcal{H}_{n}, h^{\prime}(x)=0^{n-t(n)} \| h(x)_{1, \ldots, t(n)}\right\}\right\}$, is an efficient pairwise-independent function family ensemble, mapping strings of length $n$ to the set $[T(n)]_{\{0,1\}^{n}}$.

\subsubsection{Pseudorandom Functions}

Definition 2.5 (pseudorandom functions). An efficient function family ensemble $\mathcal{F}=$ $\left\{\mathcal{F}_{n}:\{0,1\}^{n} \mapsto\{0,1\}^{\ell(n)}\right\}_{n \in \mathbb{N}}$ is a $(T(n), \varepsilon(n))$-adaptive PRF, if for every oracle-aided algorithm (distinguisher) $\mathrm{D}$ of running time $T(n)$ and large enough $n$, it holds that

$$
\left|\operatorname{Pr}_{f \leftarrow \mathcal{F}_{n}}\left[\mathrm{D}^{f}\left(1^{n}\right)=1\right]-\operatorname{Pr}_{\pi \leftarrow \Pi_{n}}\left[\mathrm{D}^{\pi}\left(1^{n}\right)=1\right]\right| \leq \varepsilon(n),
$$

where $\Pi_{n}$ is the set of all functions from $\{0,1\}^{n}$ to $\{0,1\}^{\ell(n)}$. If we limit $\mathrm{D}$ above to be non-adaptive (i.e., it has to write all his oracle calls before making the first call), then $\mathcal{F}$ is called $(T(n), \varepsilon(n))$ non-adaptive PRF.

The ensemble $\mathcal{F}$ is a $t$-adaptive $P R F$, if it is a $(t, 1 / t)$-adaptive PRF according to the above definition. It is polynomially secure adaptive PRF (for short, adaptive PRF), if it is a p-adaptive PRF for every $p \in$ poly. Finally, it is super-polynomial secure adaptive PRF, if it T-adaptive PRF for some $T(n) \in n^{\omega(1)}$. The same conventions are also used for non-adaptive PRFs.

Clearly, a super-polynomial secure PRF is also polynomially secure. In Appendix A we prove that the converse is also true: a polynomially secure PRF is also super-polynomial secure PRF.

\section{Our Construction}

In this section we present the main contribution of this paper - a direct construction of an adaptive pseudorandom function family from a non-adaptive one.

Theorem 3.1 (restatement of Theorem 1.1). Let $T$ be a polynomial-time computable integer function, let $\mathcal{H}=\left\{\mathcal{H}_{n}:\{0,1\}^{n} \mapsto[T(n)]_{\{0,1\}^{n}}\right\}$ be an efficient pairwise independent function

\footnotetext{
${ }^{4}$ For our applications, see Section 3 , we can always consider $T^{\prime}(n)=2^{\lfloor\log (T(n))\rfloor}$, which only causes us a factor of two loss in the resulting security.
} 
family ensemble, and let $\mathcal{F}=\left\{\mathcal{F}_{n}:\{0,1\}^{n} \mapsto\{0,1\}^{\ell(n)}\right\}$ be a $(p(n) \cdot T(n), \varepsilon(n))$-non-adaptive PRF, where $p \in$ poly is determined by the computation time of $T, \mathcal{F}$ and $\mathcal{H}$. Then $\mathcal{F} \circ \mathcal{H}$ is a $\left(s(n), \varepsilon(n)+\frac{s(n)^{2}}{T(n)}\right)$-adaptive PRF for every $s(n)<T(n)$.

Theorem 3.1 yields the following simpler statement.

Corollary 3.2. Let $T, p$ and $\mathcal{H}$ be as in Theorem 3.1. Assuming $\mathcal{F}$ is a $(p(n) T(n))$-non-adaptive $P R F$, then $\mathcal{F} \circ \mathcal{H}$ is a $(\sqrt[3]{T(n)} / 2)$-adaptive PRF.

Proof. Applying Theorem 3.1 with respect to $s(n)=\sqrt[3]{T(n)} / 2$ and $\varepsilon(n)=\frac{1}{p(n) T(n)}$, yields that $\mathcal{F} \circ \mathcal{H}$ is a $\left(s(n), \frac{1}{p(n) T(n)}+\frac{s(n)^{2}}{T(n)}\right)$-adaptive PRF. Since $\frac{1}{p(n) T(n)}<\frac{1}{2 s(n)}$ and $\frac{s(n)^{2}}{T(n)} \leq \frac{1}{2 s(n)}$, it follows that $\mathcal{F} \circ \mathcal{H}$ is a $(s, 1 / s)$-adaptive $\mathrm{PRF}$.

To prove Theorem 3.1, we use the (non efficient) function family ensemble $\Pi \circ \mathcal{H}$, where $\Pi=\Pi_{\ell}$ (i.e., the ensemble of all functions from $\{0,1\}^{n}$ to $\{0,1\}^{\ell}$ ), and $\ell=\ell(n)$ is the output length of $\mathcal{F}$. We first show that $\mathcal{F} \circ \mathcal{H}$ is computationally indistinguishable from $\Pi \circ \mathcal{H}$, and complete the proof showing that $\Pi \circ \mathcal{H}$ is statistically indistinguishable from $\Pi$.

\section{1 $\mathcal{F} \circ \mathcal{H}$ is Computationally Indistinguishable From $\Pi \circ \mathcal{H}$}

Lemma 3.3. Let $T, \mathcal{F}$ and $\mathcal{H}$ be as in Theorem 3.1. Then for every oracle-aided distinguisher $\mathrm{D}$ of running time $T$, there exists a non-adaptive oracle-aided distinguisher $\widehat{\mathrm{D}}$ of running time $p(n) \cdot T(n)$, for some $p \in$ poly (determined by the computation time of $T, \mathcal{F}$ and $\mathcal{H}$ ), with

$$
\left|\operatorname{Pr}_{g \leftarrow \mathcal{F}_{n}}\left[\widehat{\mathrm{D}}^{g}\left(1^{n}\right)=1\right]-\operatorname{Pr}_{g \leftarrow \Pi_{n}}\left[\widehat{\mathrm{D}}^{g}\left(1^{n}\right)=1\right]\right|=\left|\operatorname{Pr}_{g \leftarrow \mathcal{F}_{n} \circ \mathcal{H}_{n}}\left[\mathrm{D}^{g}\left(1^{n}\right)=1\right]-\operatorname{Pr}_{g \leftarrow \Pi_{n} \circ \mathcal{H}_{n}}\left[\mathrm{D}^{g}\left(1^{n}\right)=1\right]\right|
$$

for every $n \in \mathbb{N}$, where $\Pi_{n}$ is the set of all functions from $\{0,1\}^{n}$ to $\{0,1\}^{\ell(n)}$.

In particular, the pseudorandomness of $\mathcal{F}$ yields that $\mathcal{F} \circ \mathcal{H}$ is computationally indistinguishable from the ensemble $\left\{\Pi_{n} \circ \mathcal{H}_{n}\right\}_{n \in \mathbb{N}}$ by an adaptive distinguisher of running time $T$.

Proof. The distinguisher $\widehat{\mathrm{D}}$ is defined as follows:

Algorithm $3.4(\widehat{\mathrm{D}})$.

Input: $1^{n}$.

Oracle: a function $\phi$ over $\{0,1\}^{n}$.

1. Compute $\phi(x)$ for every $x \in[T(n)]_{\{0,1\}^{n}}$.

2. Set $g=\phi \circ h$, where $h$ is uniformly chosen in $\mathcal{H}_{n}$.

3. Emulate $\mathrm{D}^{g}\left(1^{n}\right)$ : answer a query $x$ to $\phi$ made by $\mathrm{D}$ with $g(x)$, using the information obtained in Step 1.

Note that $\widehat{\mathrm{D}}$ makes $T(n)$ non-adaptive queries to $\phi$, and it can be implemented to run in time $p(n) T(n)$, for large enough $p \in$ poly. We conclude the proof by observing that in case $\phi$ is uniformly drawn from $\mathcal{F}_{n}$, the emulation of $\mathrm{D}$ done in $\widehat{\mathrm{D}}^{\phi}$ is identical to a random execution of $\mathrm{D}^{g}$ with $g \leftarrow \mathcal{F}_{n} \circ \mathcal{H}_{n}$. Similarly, in case $\phi$ is uniformly drawn from $\Pi_{n}$, the emulation is identical to a random execution of $\mathrm{D}^{\pi}$ with $\pi \leftarrow \Pi_{n}$. 


\section{$3.2 \Pi \circ \mathcal{H}$ is Statistically Indistinguishable From $\Pi$}

The following lemma is commonly used for proving the security of hash based MACs (cf., [9, Proposition 6.3.6]), yet for completeness we give it a full proof below.

Lemma 3.5. Let $n, T$ be integers with $T \leq 2^{n}$, and let $\mathcal{H}$ be a pairwise-independent function family mapping string of length $n$ to $[T]_{\{0,1\}^{n}}$. Let $\mathrm{D}$ be an (unbounded) s-query oracle-aided algorithm (i.e., making at most $s$ queries), then

$$
\left|\operatorname{Pr}_{g \leftarrow \Pi \circ \mathcal{H}}\left[\mathrm{D}^{g}=1\right]-\operatorname{Pr}_{\pi \leftarrow \Pi}\left[\mathrm{D}^{\pi}=1\right]\right| \leq s^{2} / T,
$$

where $\Pi$ is the set of all functions from $\{0,1\}^{n}$ to $\{0,1\}^{\ell}$ (for some $\ell \in \mathbb{N}$ ).

Proof. We assume for simplicity that $\mathrm{D}$ is deterministic (the reduction to the randomized case is standard) and makes exactly $s$ valid (i.e., inside $\left.\{0,1\}^{n}\right)$ distinct queries, and let $\Omega=\left(\{0,1\}^{\ell}\right)^{s}$. Consider the following random process:

\section{Algorithm 3.6.}

1. Emulate D, while answering the $i$ 'th query $q_{i}$ with a uniformly chosen $a_{i} \in\{0,1\}^{\ell}$. Set $\bar{q}=\left(q_{1}, \ldots, q_{s}\right)$ and $\bar{a}=\left(a_{1}, \ldots, a_{s}\right)$.

2. Choose $h \leftarrow \mathcal{H}$.

3. Emulate D again, while answering the $i$ 'th query $q_{i}^{\prime}$ with $a_{i}^{\prime}=a_{i}$ (the same $a_{i}$ from Step 1), if $h\left(q_{i}^{\prime}\right) \notin\left\{h\left(q_{j}^{\prime}\right)\right\}_{j \in[i-1]}$, and with $a_{i}^{\prime}=a_{j}$, if $h\left(q_{i}^{\prime}\right)=h\left(q_{j}^{\prime}\right)$ for some $j \in[i-1]$.

Set $\overline{q^{\prime}}=\left(q_{1}^{\prime}, \ldots, q_{s}^{\prime}\right)$ and $\overline{a^{\prime}}=\left(a_{1}^{\prime}, \ldots, a_{s}^{\prime}\right)$.

Let $\bar{A}, \bar{Q}, \overline{A^{\prime}}, \overline{Q^{\prime}}$ and $H$ be the (jointly distributed) random variables induced by the values of $\bar{q}, \bar{a}, \overline{q^{\prime}}, \overline{a^{\prime}}$ and $h$ respectively, in a random execution of the above process. It is not hard to verify that $\bar{A}$ is distributed the same as the oracle answers in a random execution of $\mathrm{D}^{\pi}$ with $\pi \leftarrow \Pi$, and that $\overline{A^{\prime}}$ is distributed the same as the oracle answers in a random execution of $\mathrm{D}^{g}$ with $g \leftarrow \Pi \circ \mathcal{H}$. Hence, for proving Lemma 3.5, it suffices to bound the statistical distance between $\bar{A}$ and $\overline{A^{\prime}}$.

Let Coll be the event that $H\left(\bar{Q}_{i}\right)=H\left(\bar{Q}_{j}\right)$ for some $i \neq j \in[s]$. Since the queries and answers in both emulations of 3.6 are the same until a collision with respect to $H$ occurs, it follows that

$$
\operatorname{Pr}\left[\bar{A} \neq \overline{A^{\prime}}\right] \leq \operatorname{Pr}[\text { Coll }]
$$

On the other hand, since $H$ is chosen after $\bar{Q}$ is set, the pairwise independent of $\mathcal{H}$ yields that

$$
\operatorname{Pr}[\text { Coll }] \leq s^{2} / T
$$

and therefore $\operatorname{Pr}\left[\bar{A} \neq \overline{A^{\prime}}\right] \leq s^{2} / T$. It follows that $\operatorname{Pr}[\bar{A} \in C] \leq \operatorname{Pr}\left[\overline{A^{\prime}} \in C\right]+s^{2} / T$ for every $C \subseteq \Omega$, yielding that $\operatorname{SD}\left(\bar{A}, \overline{A^{\prime}}\right) \leq s^{2} / T$. 


\subsection{Putting It Together}

We are now finally ready to prove Theorem 3.1.

Proof of Theorem 3.1. Let D be an oracle-aided algorithm of running time $s$ with $s(n)<T(n)$. Lemma 3.3 yields that $\left|\operatorname{Pr}_{g \leftarrow \mathcal{F}_{n} \circ \mathcal{H}_{n}}\left[\mathrm{D}^{g}\left(1^{n}\right)=1\right]-\operatorname{Pr}_{g \leftarrow \Pi_{n} \circ \mathcal{H}_{n}}\left[\mathrm{D}^{g}\left(1^{n}\right)=1\right]\right| \leq \varepsilon(n)$ for large enough $n$, where Lemma 3.5 yields that $\left|\operatorname{Pr}_{g \leftarrow \Pi_{n} \circ \mathcal{H}_{n}}\left[\mathrm{D}^{g}\left(1^{n}\right)=1\right]-\operatorname{Pr}_{\pi \leftarrow \Pi_{n}}\left[\mathrm{D}^{\pi}\left(1^{n}\right)=1\right]\right| \leq$ $s(n)^{2} / T(n)$ for every $n \in \mathbb{N}$. Hence, the triangle inequality yields that $\left|\operatorname{Pr}_{g \leftarrow \mathcal{F}_{n} \circ \mathcal{H}_{n}}\left[\mathrm{D}^{g}\left(1^{n}\right)=1\right]-\operatorname{Pr}_{\pi \leftarrow \Pi_{n}}\left[\mathrm{D}^{\pi}\left(1^{n}\right)=1\right]\right| \leq \varepsilon(n)+s(n)^{2} / T(n)$ for large enough $n$, as requested.

\subsection{Handling Polynomial Security}

Corollary 3.2 is only useful when the security of the underlying non-adaptive PRF (i.e., $T$ ) is efficiently computable (or when considering non-uniform PRF constructions, see Section 1.1). In this section we show how to handle the important case of polynomially secure non-adaptive PRF. We use the following "combiner".

Definition 3.7. Let $\mathcal{H}$ be a function family into $\{0,1\}^{n}$. For $i \in[n]$, let $\widehat{\mathcal{H}}^{i}$ be the function family $\widehat{\mathcal{H}}^{i}=\{\widehat{h}: h \in \mathcal{H}\}$, where $\widehat{h}(x)=0^{n-i} \| h(x)_{1, \ldots, i}$.

Corollary 3.8. Let $\mathcal{F}$ be a $T(n)$-non-adaptive $P R F$, let $\mathcal{H}$ be an efficient length-preserving pairwiseindependent function family ensemble, and let $\mathcal{I}(n) \subseteq[n]$ be polynomial-time computable (in $n$ ) index set. Define the function family ensemble $G=\left\{G_{n}\right\}_{n \in \mathbb{N}}$, where $G_{n}=\bigoplus_{i \in \mathcal{I}(n)}\left(\mathcal{F}_{n} \circ \widehat{\mathcal{H}}_{n}^{i}\right)$.

There exists $q \in$ poly such that $G$ is a $\left(\sqrt[3]{2^{t(n)}} / 2\right)$-adaptive PRF, for every polynomial-time computable integer function $t$, with $t(n) \in \mathcal{I}(n)$ and $2^{t(n)} \leq T(n) / q(n)$.

Before proving the corollary, let us first use it for constructing adaptive PRF from non-adaptive polynomially secure one.

Corollary 3.9 (restatement of Corollary 1.2). Let $\mathcal{F}$ be a polynomially secure non-adaptive $P R F$, let $\mathcal{H}$ be an efficient pairwise-independent length-preserving function family ensemble and let $k(n) \in \omega(1)$ be polynomial-time computable function. Then $G:=\left\{\bigoplus_{i \in[k(n)]}\left(\mathcal{F}_{n} \circ \widehat{\mathcal{H}}_{n}^{\lfloor i \cdot \log n\rfloor}\right)\right\}_{n \in \mathbb{N}}$ is polynomially secure adaptive PRF.

Proof. Let $\mathcal{I}(n):=\{\lfloor\log n\rfloor,\lfloor 2 \cdot \log n\rfloor \ldots,\lfloor k(n) \cdot \log n\rfloor\}$. Applying Corollary 3.8 with respect to $\mathcal{F}, \mathcal{H}, \mathcal{I}$ and $t(n)=\lfloor c \cdot \log n\rfloor$, where $c \in \mathbb{N}$, yields that $G$ is a $O\left(\sqrt[3]{n^{c}}\right)$-adaptive PRF. It follows that $G$ is $p$-adaptive PRF for every $p \in$ poly. Namely, $G$ is polynomially secure adaptive PRF.

Remark 3.10 (unknown security). Corollary 3.8 is also useful when the security of $\mathcal{F}$ is "not known" in the construction time. Taking $\mathcal{I}(n)=\left\{1,2,4, \ldots, 2^{\lfloor\log n\rfloor}\right\}$ (resulting in $\log n$ calls to $\mathcal{F}$ ) and assuming that $\mathcal{F}$ is found to be $T(n)$-non-adaptive PRF for some polynomial-time computable $T$, the resulting PRF is guaranteed to be $O(\sqrt[6]{T(n)})$-adaptive PRF (neglecting polynomial factors).

Proof of Corollary 3.8. It is easy to see that $G$ is efficient, so it is left to argue for its security. Let $q(n)=q^{\prime}(n) p(n)$, where $p$ is as in the statement of Corollary 3.2, and $q^{\prime} \in$ poly to be determined later. Let $t$ be a polynomial-time computable integer function with $t(n) \in \mathcal{I}(n)$ and $2^{t(n)} \leq$ 
$T(n) / q(n)$. It follows that $\widehat{\mathcal{H}}^{t}=\left\{\widehat{\mathcal{H}}_{n}^{t(n)}\right\}_{n \in \mathbb{N}}$ is an efficient pairwise-independent function family ensemble, and Corollary 3.2 yields that $\mathcal{F} \circ \widehat{\mathcal{H}}^{t}$ is a $\left(\sqrt[3]{q^{\prime}(n) 2^{t(n)}} / 2\right)$-adaptive PRF.

Assume towards a contradiction that there exists an oracle-aided distinguisher $\mathrm{D}$ that runs in time $T^{\prime}(n)=\sqrt[3]{2^{t(n)}} / 2$ and

$$
\left|\operatorname{Pr}_{g \leftarrow G_{n}}\left[\mathrm{D}^{g}\left(1^{n}\right)=1\right]-\operatorname{Pr}_{\pi \leftarrow \Pi_{n}}\left[\mathrm{D}^{\pi}\left(1^{n}\right)=1\right]\right|>1 / T^{\prime}(n)
$$

for infinitely many $n$ 's. We use the following distinguisher for breaking the pseudorandomness of $\mathcal{F} \circ \widehat{\mathcal{H}}^{t}$ :

Algorithm 3.11 ( $\widehat{D})$.

Input: $1^{n}$.

Oracle: a function $\phi$ over $\{0,1\}^{n}$.

1. For every $i \in \mathcal{I}(n) \backslash\{t(n)\}$, choose $g^{i} \leftarrow \mathcal{F}_{n} \circ \widehat{\mathcal{H}}_{n}^{i}$.

2. Set $g:=\phi \oplus \bigoplus_{i \in \mathcal{I}(n) \backslash\{t(n)\}} g^{i}$.

3. Emulate $\mathrm{D}^{g}\left(1^{n}\right)$.

Note that $\widehat{\mathrm{D}}$ can be implemented to run in time $|\mathcal{I}(n)| \cdot r(n) \cdot T^{\prime}(n)$ for some $r \in$ poly, which is smaller than $\sqrt[3]{q^{\prime}(n) 2^{t(n)}} / 2$ for large enough $q^{\prime}$. Also note that in case $\phi$ is uniformly distributed over $\Pi_{n}$, then $g$ (selected by $\widehat{\mathrm{D}}^{\phi}\left(1^{n}\right)$ ) is uniformly distributed in $\Pi_{n}$, where in case $\phi$ is uniformly distributed in $\mathcal{F}_{n} \circ \widehat{\mathcal{H}}_{n}^{t(n)}$, then $g$ is uniformly distributed in $G_{n}$. It follows that

$$
\left|\operatorname{Pr}_{g \leftarrow\left(\mathcal{F} \circ \widehat{\mathcal{H}}^{t}\right)_{n}}\left[\widehat{\mathrm{D}}^{g}\left(1^{n}\right)=1\right]-\operatorname{Pr}_{\pi \leftarrow \Pi_{n}}\left[\widehat{\mathrm{D}}^{\pi}\left(1^{n}\right)=1\right]\right|=\left|\operatorname{Pr}_{g \leftarrow G_{n}}\left[\mathrm{D}^{g}\left(1^{n}\right)=1\right]-\operatorname{Pr}_{\pi \leftarrow \Pi_{n}}\left[\mathrm{D}^{\pi}\left(1^{n}\right)=1\right]\right|
$$

for every $n \in \mathbb{N}$. In particular, Equation (3) yields that

$$
\left|\operatorname{Pr}_{g \leftarrow\left(\mathcal{F} \circ \widehat{\mathcal{H}}^{t}\right)_{n}}\left[\widehat{\mathrm{D}}^{g}\left(1^{n}\right)=1\right]-\operatorname{Pr}_{\pi \leftarrow \Pi_{n}}\left[\widehat{\mathrm{D}}^{\pi}\left(1^{n}\right)=1\right]\right|>\frac{2}{\sqrt[3]{2^{t(n)}}}>\frac{2}{\sqrt[3]{q^{\prime}(n) 2^{t(n)}}}
$$

for infinitely many $n$ 's, in contradiction to the pseudorandomness of $\mathcal{F} \circ \widehat{\mathcal{H}}^{t}$ we proved above.

\section{Acknowledgment}

We are very grateful to Omer Reingold for very useful discussions, and for challenging the second author with this research question a long while ago. 


\section{References}

[1] M. Bellare. A note on negligible functions. Journal of Cryptology, pages 271-284, 2002.

[2] M. Bellare and S. Goldwasser. New paradigms for digital signatures and message authentication based on non-interative zero knowledge proofs. In Advances in Cryptology - CRYPTO '89, pages 194-211, 1989.

[3] M. Blum, W. S. Evans, P. Gemmell, S. Kannan, and M. Naor. Checking the correctness of memories. Algorithmica, 12(2/3):225-244, 1994.

[4] L. J. Carter and M. N. Wegman. Universal classes of hash functions. Journal of Computer and System Sciences, pages 143-154, 1979.

[5] C. Cho, C.-K. Lee, and R. Ostrovsky. Equivalence of uniform key agreement and composition insecurity. In Advances in Cryptology - CRYPTO 2010, pages 447-464, 2010.

[6] B. Chor, A. Fiat, M. Naor, and B. Pinkas. Tracing traitors. IEEE Transactions on Information Theory, 46(3):893-910, 2000.

[7] O. Goldreich. Towards a theory of software protection. In Advances in Cryptology - CRYPTO '86, pages 426-439, 1986.

[8] O. Goldreich. Foundations of Cryptography: Basic Tools. Cambridge University Press, 2001.

[9] O. Goldreich. Foundations of Cryptography - VOLUME 2: Basic Applications. Cambridge University Press, 2004.

[10] O. Goldreich, S. Goldwasser, and S. Micali. On the cryptographic applications of random functions. pages 276-288, 1984.

[11] O. Goldreich, S. Goldwasser, and S. Micali. How to construct random functions. Journal of the ACM, pages 792-807, 1986.

[12] J. Håstad, R. Impagliazzo, L. A. Levin, and M. Luby. A pseudorandom generator from any one-way function. SIAM Journal on Computing, pages 1364-1396, 1999.

[13] M. Luby. Pseudorandomness and cryptographic applications. Princeton computer science notes. Princeton University Press, 1996. ISBN 978-0-691-02546-9.

[14] M. Luby and C. Rackoff. How to construct pseudorandom permutations from pseudorandom functions. SIAM Journal on Computing.

[15] U. M. Maurer and K. Pietrzak. Composition of random systems: When two weak make one strong. In Theory of Cryptography, First Theory of Cryptography Conference, TCC 2004, pages 410-427, 2004.

[16] S. Myers. Black-box composition does not imply adaptive security. In Advances in Cryptology - EUROCRYPT 2004, pages 189-206, 2004. 
[17] M. Naor and O. Reingold. Synthesizers and their application to the parallel construction of psuedo-random functions. In Proceedings of the 36th Annual Symposium on Foundations of Computer Science (FOCS), pages 170-181, 1995.

[18] R. Ostrovsky. An efficient software protection scheme. In Advances in Cryptology - CRYPTO '89, 1989.

[19] K. Pietrzak. Composition does not imply adaptive security. In Advances in Cryptology CRYPTO 2005, pages 55-65, 2005.

[20] K. Pietrzak. Composition implies adaptive security in minicrypt. In Advances in Cryptology - EUROCRYPT 2006, pages 328-338, 2006.

\section{A From Polynomial to Super-Polynomial Security}

The standard security definition for cryptographic primitives is polynomial security: any PPT trying to break the primitive has only negligible success probability. Bellare [1] showed that for any polynomially secure primitive there exists a single negligible function $\mu$, such that no PPT can break the primitive with probability larger than $\mu$. Here we take his approach a step further, showing that for a polynomially secure primitive there exists a super-polynomial function $T$, such that no adversary of running time $T$ breaks the primitive with probability larger than $1 / T$.

In the following we identify algorithms with their string description. In particular, when considering algorithm $A$, we mean the algorithm defined by the string $A$ (according to some canonical representation). We prove the following result.

Theorem A.1. Let $v:\{0,1\}^{*} \times \mathbb{N} \mapsto[0,1]$ be a function with the following properties: 1) $v(\mathrm{~A}, n) \leq$ $1 / p(n)$ for every oracle-aided PPT A, $p \in$ poly and large enough $n$; and 2) if the distributions induced by random executions of $\mathrm{A}^{f}(x)$ and $\mathrm{B}^{f}(x)$ are the same for any input $x \in\{0,1\}^{n}$ and function $f$ (each distribution describes the algorithm's output and oracle queries), then $v(\mathrm{~A}, n)=v(\mathrm{~B}, n)$.

Then there exists an integer function $T(n) \in n^{\omega(1)}$ such that following holds: for any algorithm A of running time at most $T(n)$, it holds that $v(\mathrm{~A}, n) \leq 1 / T(n)$ for large enough $n$.

Remark A.2 (Applications). Let $f$ be a polynomially secure OWF (i.e., $\operatorname{Pr}\left[\mathrm{A}\left(f\left(U_{n}\right)\right) \in\right.$ $\left.f^{-1}\left(f\left(U_{n}\right)\right)\right]=\operatorname{neg}(n)$ for any $\left.\mathrm{PPT} \mathrm{A}\right)$. Applying Theorem A.1 with $v(\mathrm{~A}, n):=\operatorname{Pr}\left[\mathrm{A}\left(f\left(U_{n}\right)\right) \in\right.$ $\left.f^{-1}\left(f\left(U_{n}\right)\right)\right]$ (where if $\mathrm{A}$ expects to get an oracle, provide him with the constant function $\phi(x)=1$ ), yields that $f$ is super-polynomial secure $O W F$ (i.e., exists $T(n) \in n^{\omega(1)}$ such that $\operatorname{Pr}\left[\mathrm{A}\left(f\left(U_{n}\right)\right) \in\right.$ $\left.f^{-1}\left(f\left(U_{n}\right)\right)\right] \leq 1 / T(n)$ for any algorithm of running time $T$ and large enough $\left.n\right)$.

Similarly, for a polynomially secure PRF $\mathcal{F}=\left\{\mathcal{F}_{n}\right\}_{n \in \mathbb{N}}$ (see Definition 2.5), applying Theorem A.1 with $v(\mathrm{~A}, n):=\left|\operatorname{Pr}_{f \leftarrow \mathcal{F}_{n}}\left[A^{f}\left(1^{n}\right)=1\right]-\operatorname{Pr}_{\pi \leftarrow \Pi_{n}}\left[A^{\pi}\left(1^{n}\right)=1\right]\right|$, where $\Pi_{n}$ is the set of all functions with the same domain/range as $\mathcal{F}_{n}$, yields that $\mathcal{F}$ is super-polynomial secure PRF.

Proof of Theorem A.1. Given a probabilistic algorithm $\mathrm{A}$ and an integer $i$, let $\mathrm{A}_{i}$ denote the variant of A that on input of length $n$, halts after $n^{i}$ steps (hence, $\mathrm{A}_{i}$ is a PPT). Let $\mathcal{S}_{i}$ be the first $i$ strings in $\{0,1\}^{*}$, according to some canonical order, viewed as descriptions of $i$ algorithms. Let $\mathcal{I}(n)=\left\{i \in[n]: \forall \mathrm{A} \in \mathcal{S}_{i}, k \geq n: v\left(\mathrm{~A}_{i}, k\right)<1 / k^{i}\right\} \cup\{1\}$, let $t(n)=\max \mathcal{I}(n)$ and $T(n)=n^{t(n)}$.

Let $\mathrm{A}$ be an algorithm of running time $T(n)$, and let $i_{\mathrm{A}}$ be the first integer such that $\mathrm{A} \in \mathcal{S}_{i_{\mathrm{A}}}$. In Claim A.3 we prove that $t(n) \in \omega(1)$, hence it follows that $t(n)>i_{\mathrm{A}}$ for any large enough 
$n$. For any such $n$, the definition of $t$ guarantees that $v\left(\mathrm{~A}_{t(n)}, n\right)<1 / n^{t(n)}=1 / T(n)$. Since $\mathrm{A}$ is of running time $T(n)$, the second property of $v$ yields that $v(\mathrm{~A}, n)=v\left(\mathrm{~A}_{t(n)}, n\right)$, and therefore $v(\mathrm{~A}, n)<1 / T(n)$.

Claim A.3. It holds that $t(n) \in \omega(1)$.

Proof. Fix $i \in \mathbb{N}$. For each $\mathrm{A} \in \mathcal{S}_{i}$, let $n_{\mathrm{A}}$ be the first integer such that $v\left(\mathrm{~A}_{i}, n\right) \leq 1 / n^{i}$ for every $n \geq n_{\mathrm{A}}$ (note that such $n_{\mathrm{A}}$ exists by the first property of $v$ ), and let $n_{i}=\max \left\{n_{\mathrm{A}}: \mathrm{A} \in \mathcal{S}_{i}\right\}$. It follows that $v\left(\mathrm{~A}_{i}, n\right) \leq 1 / n^{i}$ for every $n \geq n_{i}$ and $\mathrm{A} \in \mathcal{S}_{i}$, and therefore $t\left(n_{i}\right) \geq i$. 